\title{
Plasmid-Mediated Resistance to Extended-Spectrum Cephalosporins and Resistance to Fluoroquinolones in Escherichia Coli Isolates from Black- headed Gulls (Larus Ridibundus)
}

Maja Velhner ( $\nabla$ maja@niv.ns.ac.rs )

Scientific Veterinary Institute Novi Sad

Dalibor Todorović

Scientific Veterinary Institute "Novi Sad", Novi Sad, Serbia

\section{Katarina Novović}

Institute of Molecular Genetics and Genetic Engineering, University of Belgrade, Belgrade, Serbia

\section{Branko Jovčić}

Institute of Molecular genetics and Genetic Engineering, University of Belgrade, Belgrade, Serbia, Faculty of Biology, University of Belgrade, Belgrade, Serbia

Gospava Lazić

Scientific Veterinary Institute "Novi Sad, Novi Sad, Serbia

Milan Kojić

Institute of Molecular Genetics and Genetic Engineering, University of Belgrade, Serbia

Corinna Kehrenberg

Institut fur Tierarztliche Nahrungsmitteelkunde, Justus-Liebig-Universitat Giessen, Germany

Original Article

Keywords: Escherichia coli, gulls, ESC, FQ, plasmid

Posted Date: January 29th, 2021

DOI: https://doi.org/10.21203/rs.3.rs-157826/v1

License: @ (i) This work is licensed under a Creative Commons Attribution 4.0 International License. Read Full License 


\section{Abstract}

Although resistance to fluoroquinolones is common in $E$. coli isolates from farm and game animals in Serbia, currently no data are accessible on the occurrence of antibacterial resistances in $E$. coli isolates from gulls. Therefore, 45 cloacal swabs and 50 fecal samples from black-headed gulls were investigated for the presence of Escherichia coli isolates resistant to antibiotics. Multidrug resistance was detected in $22 \mathrm{E}$. coli isolates. High level resistance to fluoroquinolones was found in ten isolates with MIC values of ciprofloxacin ranging from 4 to $32 \mathrm{mg} / \mathrm{L}$. Genotyping revealed single or double mutations in the quinolone resistance determining region (QRDR) of the gyrA or gyrA, parC and parE genes, respectively. Ten isolates showed resistance to extendedspectrum cephalosporin antibiotics. These ten isolates belonged to phylogenetic group B2 (five isolates), group D (four isolates) and group B1 (one isolate). An extended-spectrum $\beta$-lactamase resistance phenotype was detected in one isolate which carried the $b / a_{\mathrm{CTX}-\mathrm{M}-1}$ gene on a plasmid of the I2/FIB replicon type. Nine isolates carried bla $a_{\mathrm{CMY}-2}$ genes, which were detected on conjugative plasmids in seven isolates. One transconjugant also carried $h / y$, iroN, iss, ompT and $c v a C$ virulence genes on the plasmid. Five different sequence types (ST38, ST2307, ST224, ST162 and ST34) were detected in E. coli isolates with ESBL or AmpC phenotype and genotype.

\section{Introduction}

The escalating development of antimicrobial resistance in pathogenic and commensal bacteria is a well-known worldwide problem. There are several ways in which bacteria can become resistant to antibiotics including point mutations within the bacterial chromosome, efflux mechanisms and reduced permeability or uptake of antimicrobials, enzymatic inactivation or alterations of the target site (Adamassie, 2018). Genes mediating antimicrobial resistance are often found inside mobile genetic elements such as plasmids, transposons, integrons and insertion sequences. This complex genetic material can be easily transferred between bacterial species which also facilitates the spread of resistant bacteria in the environment (Levy and Marchall, 2004; Wellington et al., 2013; Vandecraen et al., 2017). Pathogenic and multidrug-resistant bacteria can thus become widespread pathogens in hospitals or prevalent on production plants. In this case, the only way to reduce contamination and spread is to use antibiotics prudently and continuously apply the best standards of hygiene. On the other hand, it is an extremely challenging venture to reduce virulent and multidrug-resistant bacteria from the environment and wildlife.

Escherichia coli isolates from wild birds, mostly gulls, have been the focus of various studies and it was shown that wild birds carry bacteria that are often resistant to numerous antibiotics (Parker et al., 2016; Zurfluh et al., 2019). Multidrug-resistant $E$. coli have been identified in gulls residing in remote parts of the world (Hernandez et al., 2013; Ramey et al., 2018), on coastlines (Poirel et al., 2012 and beaches (Simões et al., 2010) worldwide. However, it is also important to note that in some cases mobile resistance genes have been detected in virulent $E$. coli from wildlife, but that these may have originated from humans or farm animals (Hernandez et al., 2013; Atterby et al., 2016; Atterby et al., 2017; Ahlstrom et al., 2018). In addition, a de novo development of resistance in environmental pathogens can also occur (e.g. to synthetic antibiotics such as fluoroquinolones), which can result from residues and contamination of habitats as a consequence of anthropogenic activities. This is supported by the fact that fluoroquinolones can accumulate in natural environments, especially in soil and water (Grenni et al., 2018).

It is important to note that resistance to fluoroquinolones has been detected in multidrug-resistant $E$. coli isolates from cattle, pigs and wild animals in Serbia (Todorović et al., 2018; Velhner et al., 2018). This type of resistance may be established also in $E$. coli isolates from wild birds which may facilitate the dissemination of fluoroquinolone-resistant $E$. coli in the environment.

Extended-spectrum $\beta$-lactamase-producing E. coli (ESBL) is frequently detected in the livestock industry in both diarrheic and healthy animals (Faccone et al., 2019; Shabana and Al-Enazi, 2020). Often ESBL-producing E. coli is also resistant to fluoroquinolones leaving fewer options for treatment of infections caused by the resistant bacteria in both human and veterinary medicine. Even well-established clones in humans, such as E. coli ST131-CTX-15, are occasionally detected in animals or the environment worldwide (Johnson et al., 2012; Nicolas-Chamoine et al., 2013). The adaptation of ESBL-producing E. coli to various niches could be explained by its mechanisms of virulence and the antibiotic selection mechanisms (Nicolas-Chamoine et al., 2013).

Therefore, we analyzed the resistance mechanisms to different classes of antibiotics including fluoroquinolones in $E$. coli isolates from black-headed gulls. Besides, the plasmid-mediated resistance to cephalosporin antibiotics was studied in detail and determined whether these $E$. coli isolates harbor virulence genes on their conjugative plasmids.

\section{Material And Methods Sampling}

E. coli isolates were obtained from 45 cloacal swabs and 50 fecal samples from black-headed gulls (Larus ridibundus) collected during winter months in the city of Novi Sad in 2014. The cloacal swabs, which received the designation 1773, were taken on the local land field during a ringing campaign. For the trial, three permits were obtained, one from the Ministry of Agriculture and Environmental Protection of the Republic of Serbia (permit number 353-01-768/2014-08), one from the Ministry for Energy, Development and Environment Protection of the Republic of Serbia (permit number 353-01-845/2013-08) and one from the Institute for Nature Conservation of Vojvodina Province (permit number 1953 - 230). Twenty-four E. coli isolates from cloacal swabs that were resistant to antibiotics were included in further research. Thirty-four fecal samples were collected at a local beach ("Strand") and marked with 1774 , while 16 were collected at the pier on the Danube and marked with 1775 and 1776 . Twenty E. coli isolates obtained from feces and the 24 isolates from cloacal swabs that were resistant to antibiotics were included in the further research due to their single or multidrug-resistant phenotype.

Isolation and identification of E. coli 
E. coli were isolated by inoculating a single swab in $10 \mathrm{~mL}$ of peptone water (Oxoid, Basingstoke, Hampshire, England CM1049). After 18 hours of incubation at $37^{\circ} \mathrm{C}$, a full loop $(10 \mu \mathrm{L})$ was used to spread the inoculum over McConkey agar. Single colonies were thus collected and subsequent passages were done to obtain a pure culture. E. coli isolates were stored at $-80^{\circ} \mathrm{C}$ in tryptic soy broth with $20 \%$ glycerin until further use. Species confirmation was done by polymerase chain reaction using a protocol described by McDaniels et al. 1996 for the detection of the gadA/B gene encoding a glutamate decarboxylase in $E$. coli.

\section{Resistotyping and MIC determination of ciprofloxacin and colistin}

Resistotypes were determined as recommended by the Clinical and Laboratory Standards Institute (documents number M07 and M100, 2018). The following antibiotic disks with the recommended concentration of antibiotics were used in the study: ampicillin $10 \mu \mathrm{g}$ (AMP), amoxicillin/clavulanic acid $20 \mu \mathrm{g}+10 \mu \mathrm{g}$ (AMC), chloramphenicol $30 \mu \mathrm{g}(\mathrm{CHL})$, ciprofloxacin $5 \mu \mathrm{g}(\mathrm{CIP})$, gentamicin $10 \mu \mathrm{g}(\mathrm{GEN})$, nalidixic acid $30 \mu \mathrm{g}$ (NAL), streptomycin $10 \mu \mathrm{g}(\mathrm{STR})$, sulfonamides $300 \mu \mathrm{g}(\mathrm{SA})$, tetracycline $30 \mu \mathrm{g}(\mathrm{TET})$, trimethoprim/sulfamethoxazole 1.25 / $23.75 \mu \mathrm{g}(\mathrm{SXT})$, trimethoprim $5 \mu \mathrm{g}$ (TMP), cefpodoxime $10 \mu \mathrm{g}$ (CPD), cefotaxime $30 \mu \mathrm{g}$ (CTX), and ceftazidime $30 \mu \mathrm{g}$ (CAZ), cefoxitin $30 \mu \mathrm{g}$ (FOX). Disks were purchased from BioRad (Marnes-la-Coquette, France). For quality control, E. coli ATCC 25922 was used. Multidrug resistance was assigned to isolates that were resistant to $\geq 3$ antibiotics of different classes (Schwarz et al., 2010). Synergy tests for detection of extended-spectrum- $\beta$-lactamase producing E. coli were done as recommended in the CLSI document M100, 2018. The interpretation of phenotypic tests for the detection of plasmid-mediated pAmpC $\beta$-lactamase production included cefoxitin ( $30 \mu \mathrm{g})$ and cefepime ( $30 \mu \mathrm{g})$ disks as recommended by the EFSA Journal (2019). Testing of MICs of ciprofloxacin and colistin was done by the broth microdilution method in cation-adjusted Mueller Hinton broth (Oxoid, Basingstoke, Hampshire, England). However, MICs of colistin were determined following the protocol by Gwozdzinski et al., 2018, in Mueller Hinton broth (Sigma-Aldrich, Schnelldorf, Germany) supplemented with calcium chloride dehydrate (Roth, Karlsruhe, Germany). E. coli ATCC 25922 and E. coli NCTC 13846 were included on each plate for quality control purposes.

\section{Resistance and virulence gene screening and sequencing}

The primer sequences, annealing temperatures and references used for the resistance and virulence gene screening including primers used for phylogenetic and replicon typing, by PCR are presented in Supplementary Table 1. The master mix for beta-lactam gene detection was prepared by using a commercial kit One Taq Hot Start 2x Master Mix M0484, (New England BioLabs, Ipswich, MA, USA) and amplicons were purified using the commercial kit Monarch, PCR and DNA Clean up Kit (New England, BioLabs, Ipswich, MA, USA) and then sent to Macrogen, Amsterdam, Holland for sequencing of both strands. For sequencing the quinolone determining regions (QRDRs) of the topoisomerase genes, the Qiagen Hot Star Taq Master Mix commercial kit (Qiagen, Hilden, Germany) was used for DNA extraction followed by a DNA purification step using the QiAquick PCR purification kit of the same manufacturer. The sequences of the bla $a_{\mathrm{CMY}-2}$ and bla ${ }_{\mathrm{CTX}-\mathrm{M}-1}$ genes and of the QRDR genes were analyzed with the Basic Local Alignment Search Tool-nucleotide program-BLAST (https://blast.ncbi.nlm.nih.gov/Blast.cgi?PROGRAM=blastn).

\section{Mating experiment and S1 nuclease assay}

The isolates that were resistant to extended-spectrum cephalosporins were included in mating experiments with the recipient strain E. coli HK225. For these experiments, Luria Bertani medium (Becton Dickinson, Sparks, MD, Le Pont de Claix, France) was supplemented with 2 mg/L cefotaxime and 100 mg/L rifampicin. The isolates were shaken in the liquid medium for 30 minutes and the obtained transconjugants were used for further analysis. DNA preparation and restriction enzyme digestion for Pulsed-field Gel Electrophoresis (PFGE) of seven E. coli isolates, the recipient strain and the resulting transconjugants were performed as previously described, (Jovcic et al., 2011). The DNA was either digested with the restriction enzyme Xbal or with S1 nuclease or was not digested. PFGE was performed with a 2015 Pulsafor unit (LKB Instruments, Bromma, Sweden) equipped with a hexagonal electrode array for $18 \mathrm{~h}$ at $300 \mathrm{~V}$ at $9^{\circ} \mathrm{C}$. The gels were stained with ethidium bromide and photographed under UV illumination. Transconjugants were confirmed based on the comparisons of $X$ bal macrorestriction profiles and the presence of plasmid bands in the S1 nuclease assay. The efficiency of conjugation was estimated according to Phornphisutthimas et al. (2007).

\section{Multilocus sequence typing of transconjugants}

Multilocus sequence typing (MLST) was performed by PCR amplification and sequencing of seven housekeeping genes (adk, fumC, gyrB, icd, $m d h$, purA and recA) using primers and conditions defined at the EnteroBase E. coli MLST database (https://enterobase.readthedocs.io/en/latest/mlst/mlst-legacy-infoecoli.html) (Wirth et al., 2006). According to the allele profile, the isolates were assigned to a specific sequence type (ST) using the EnteroBase database (Supplementary Table 2). Sequence type designation was not possible for two isolates (1773/47 and 1773/64), which means that these may belong to some new sequence type and thus must be analyzed by the whole genome sequencing approach for the final MLST confirmation.

\section{Results}

\section{Multidrug resistance in E. coli isolates from black-headed gulls}

Resistance to antibiotics was detected in 44 out of $95 \mathrm{E}$. coli isolates from black-headed gulls (Table 1). Resistance to three or more antibiotics of different classes was identified in 24 isolates. However, resistance to colistin was not found (Table 1). A class 1 integron was detected only in one isolate (1773/30) which was resistant to six different antibiotics including one combination of them: AMP, CHL, STR, SA, TET, TMP/SXT. Resistance to ampicillin was most frequently detected - in 34 isolates in total- and was associated with resistance to streptomycin, sulfonamides and trimethoprim in 15 isolates. The most frequently detected gene that was responsible for resistance to beta-lactams was bla $a_{\mathrm{TEM}}$ (24 isolates), followed by bla $\mathrm{CMY}_{\mathrm{CM}-2}$ ( 9 isolates), while bla $\mathrm{CTX}_{\mathrm{M}-\mathrm{M}-1}$ was confirmed in only one isolate. The second most common resistance was found to tetracyclines, which was conferred by tet(A) or tet(B) genes alone (in 20 isolates and 4 isolates, respectively) or by both of them (2 isolates). Some $E$. coli were found to be resistant to streptomycin (20 isolates), which was mediated by the combination of strA and strB genes, which encode aminoglycoside phosphotransferases APH(3")-Ib and APH(6)-Id, respectively. 
Table 1

The resistotype and resistance gene detection in E. coli isolates from black-headed gulls

\begin{tabular}{|c|c|c|c|}
\hline Isolate No & Resistotype & $\begin{array}{l}\mathrm{MIC}(\mathrm{mg} / \mathrm{L}) \\
\mathrm{CST}\end{array}$ & Resistance genes \\
\hline $1773 / 1$ & AMP, CIP, CHL, NAL, STR, SA, TET, TMP, SXT & 0.5 & bla $a_{\mathrm{TEM}}$, cat1,strA, strB, sul2, tet(B), dfrA7/17, int1 \\
\hline $1773 / 3$ & AMP, CHL, STR, SA, TET & 0.5 & bla ${ }_{\mathrm{TEM}}$, cat1, strA, strB, sul2, tet(B) \\
\hline $1773 / 6$ & CIP, NAL, TET & 0.25 & $\operatorname{tet}(\mathrm{A})$ \\
\hline $1773 / 7$ & AMC, AMP, CPD, CAZ, CTX & 0.5 & $b / a_{\mathrm{TEM}}, b / a_{\mathrm{CMY}-2}$ \\
\hline $1773 / 21$ & AMP, STR, SA, TET, TMP, SXT & 0.5 & $b l a_{\mathrm{TEM}}, \operatorname{str} A, \operatorname{str} B, \operatorname{aad} A 2, \operatorname{sul} 2, \operatorname{tet}(\mathrm{A}), \operatorname{dfr} A 12$, int1 \\
\hline $1773 / 25$ & AMP, CIP, CHL, NAL, STR, SA, TET, TMP, SXT & 0.5 & $b l a a_{\mathrm{TEM}}, \operatorname{cat1}, \operatorname{str} A, \operatorname{str} B$, sul1, sul2, tet( $\left.\mathrm{A}\right), \operatorname{dfr} A 7 / 17$ \\
\hline $1773 / 30$ & AMP, CHL, STR, SA, TET, TMP, SXT & 0.5 & bla $a_{\mathrm{TEM}}, \operatorname{cat} 1, \operatorname{str} A, \operatorname{str} B, \operatorname{sul} 2, \operatorname{tet}(\mathrm{A}), \operatorname{dfr} A 7 / 17$, int 1 , integron 1 \\
\hline $1773 / 31$ & STR, TET & 0.5 & $s t r A, s t r B, \operatorname{tet}(B)$ \\
\hline $1773 / 40$ & TET & 0.5 & $\operatorname{tet}(\mathrm{B})$ \\
\hline $1773 / 47$ & AMC, AMP, CPD, CAZ, CTX & 0.5 & $b / a_{\mathrm{CMY}-2}$ \\
\hline $1773 / 50$ & AMC, AMP, CPD, CTX, CIP, NAL, TET & 1 & $b / a_{\mathrm{CMY}-2}, \operatorname{tet}(\mathrm{A})$ \\
\hline $1773 / 52$ & AMC, AMP, CPD, CAZ, CTX, CIP, NAL, TET & 0.5 & $b l a_{\mathrm{CMY}-2}, \operatorname{tet}(\mathrm{A})$ \\
\hline $1773 / 59$ & AMP, NAL, TET & 0.5 & $\operatorname{tet}(\mathrm{A})$ \\
\hline $1773 / 67$ & AMC, AMP, CPD, CAZ & 0.5 & $b / a_{\mathrm{CMY}-2}$ \\
\hline $1773 / 70$ & AMP, CIP, NAL, TET & 0.5 & $b l a_{\mathrm{TEM}}, \operatorname{tet}(\mathrm{A}), \operatorname{tet}(\mathrm{B})$ \\
\hline $1773 / 75$ & AMC, AMP, CPD, CAZ, CTX & 0.5 & $b / a_{\mathrm{CMY}-2}$ \\
\hline $1773 / 80$ & AMP, CPD, CTX & 0.25 & $b / a_{\mathrm{CTX}-\mathrm{M}-1}$ \\
\hline $1773 / 85$ & CHL, STR, SA, TET & 0.5 & $\operatorname{cm} / A, \operatorname{aad} A 1$, aad $A 2, \operatorname{sul} 3, \operatorname{tet}(\mathrm{A})$, int1 \\
\hline $1773 / 86$ & AMP, NAL, STR, SA, TET, TMP, SXT & 0.5 & $b l a_{\mathrm{TEM}}, \operatorname{str} A, \operatorname{str} B, \operatorname{sul} 2, \operatorname{tet}(\mathrm{A}), d f r A 5 / 14$, int 1 \\
\hline $1773 / 87 *$ & AMP, CIP, NAL, STR, SA, TET, TMP, SXT & 0.5 & $b l a_{\mathrm{TEM}}$, strA, strB, sul2, dfrA5/14 \\
\hline 4 & STR, TET & 0.5 & $\operatorname{str} A, \operatorname{str} B, \operatorname{tet}(\mathrm{A}), \operatorname{tet}(\mathrm{B})$ \\
\hline 16 & NAL, TET & 0.5 & $\operatorname{tet}(\mathrm{A})$ \\
\hline 17 & AMP, CIP, NAL, STR, SA, TET, TMP, SXT & 0.25 & bla ${ }_{\mathrm{TEM}}, \operatorname{str} A, \operatorname{str} B, a a d A 1$, sul1, sul2,tet(A),dfrA1, int1 \\
\hline $22 \mathrm{a}$ & AMC, AMP, CPD, CAZ, CTX & 1 & $b / a_{\mathrm{CMY}-2}$ \\
\hline $1774 / 1$ & AMC, AMP, CPD, CAZ, CTX, NAL, TET & 0.5 & $b / a_{\mathrm{CMY}-2}, \operatorname{tet}(\mathrm{A})$ \\
\hline $1774 / 2$ & STR, SA & 1 & strA, strB, sul2 \\
\hline $1774 / 14$ & NAL & 0.5 & / \\
\hline $1774 / 18$ & AMP, CIP, NAL, STR, SA, TET, TMP, SXT & 0.5 & $b l a_{\mathrm{TEM}}$, strA, strB, aadA1, sul1, sul2, tet(B),dfrA1 \\
\hline $1774 / 25$ & NAL & 0.5 & / \\
\hline $1774 / 29 *$ & AMP, TET & 1 & $b / a_{\mathrm{TEM}}$ \\
\hline $1774 / 37$ & AMP, STR, SA, TET, TMP, SXT & 2 & $b / a_{\mathrm{TEM}}, \operatorname{str} A, \operatorname{str} B, \operatorname{sul} 2, \operatorname{tet}(\mathrm{A}), d f r A 7 / 17$ \\
\hline $1774 / 42$ & AMP, CPD, CTX, SSS & 1 & $b / a_{\mathrm{TEM}}$, sul2 \\
\hline $1774 / 43$ & AMP, STR, SA, TET, TMP, SXT & 1 & $b l a_{\mathrm{TEM}}, \operatorname{str} A, \operatorname{str} B, s u l 2, \operatorname{tet}(\mathrm{A}), d f r A 7 / 17$ \\
\hline $1774 / 45$ & AMP & 1 & $b / a_{\mathrm{TEM}}$ \\
\hline $1775 / 17$ & AMC, AMP, CPD, CAZ, CTX & 0.5 & $b / a_{\mathrm{CMY}-2}$ \\
\hline
\end{tabular}




\begin{tabular}{|c|c|c|c|}
\hline Isolate No & Resistotype & $\begin{array}{l}\mathrm{MIC}(\mathrm{mg} / \mathrm{L}) \\
\mathrm{CST}\end{array}$ & Resistance genes \\
\hline $1775 / 20$ & AMP, STR, SA, TET & 0.5 & $b l a_{\mathrm{TEM}}, \operatorname{str} A, \operatorname{str} B, \operatorname{sul} 2, \operatorname{tet}(\mathrm{A})$ \\
\hline $1775 / 21$ & AMP,STR, SA, TET & 0.5 & $b l a_{\mathrm{TEM}}, \operatorname{str} A, \operatorname{str} B$, aadA2, sul2, $\operatorname{tet}(\mathrm{A})$ \\
\hline $1775 / 27$ & AMP,STR, SA, TET, TMP, SXT & 1 & $b l a_{\mathrm{TEM}}, \operatorname{str} A, \operatorname{str} B, \operatorname{sul} 2, \operatorname{tet}(\mathrm{A}), d f r A 5 / 14$ \\
\hline $1775 / 30$ & AMP, STR, SA, TET, TMP, SXT & 1 & $b l a \mathrm{TEM}, \operatorname{str} A, \operatorname{str} B, \operatorname{sul} 2, \operatorname{tet}(\mathrm{A}), \mathrm{dfr} A 5 / 14$ \\
\hline $1775 / 33$ & AMP,STR, SA, TET, TMP, SXT & 0.5 & bla ${ }_{\mathrm{TEM}}, \operatorname{str} A, \operatorname{str} B, \operatorname{sul} 2, \operatorname{tet}(\mathrm{A}), \operatorname{dfr} A 5 / 14$ \\
\hline $1776 / 4$ & NAL & 1 & / \\
\hline $1776 / 13$ & AMP,CIP,NAL,STR,SA, TET, TMP,SXT & 0.5 & bla ${ }_{\mathrm{TEM}}$, strA, strB, sul1, sul2, tet(A), dfrA1 \\
\hline $1776 / 19$ & AMP,SA & 0.5 & $b / a_{\mathrm{TEM}}$, sul3 \\
\hline $1776 / 28$ & AMP & 0.5 & $b / a_{\mathrm{TEM}}$ \\
\hline
\end{tabular}

Resistance to trimethoprim was detected in 14 isolates among which five carried dihydrofolate reductase genes of type $d f r A 7 / 17$ ordfrA5/14, whose products catalyse the reduction of dihydrofolate to tetrahydrofolate. Two other genes, $d f r A 1$ and $d f r A 12$, coding for dihydrofolate reductase enzymes, were detected in three and one isolates, respectively. Resistance to chloramphenicol was mediated by the gene cat1 (four isolates), which encodes a chloramphenicol acetyltransferase protein that inactivates the antibiotic by an acetylation mechanism. Another isolate carried the $\mathrm{cm} / \mathrm{A}$ gene encoding a specific transporter protein. The genes involved in resistance to sulphonamides were either sul2 (in 14 isolates) or sul3 (two isolates), while in four isolates both sul1 and sul2 genes were identified. In addition, the int1 gene coding for class 1 integrase enzymes was found in six isolates (Table 1).

\section{Characterization of E. coli isolates resistant to extended-spectrum beta-lactam antibiotics}

Ten $E$. coli isolates were resistant to extended-spectrum cephalosporin antibiotics (nine isolates were resistant to cefotaxime, ceftazidime, cefpodoxime and cefoxitin, while one isolate (1773/80) was resistant to cefotaxime and cefpodoxime (Table 2). Out of these ten isolates, three (1773/50, 1773/52, 1774/1) were co-resistant to quinolones and tetracyclines and were therefore classified as multidrug-resistant. Six of the $E$. coli isolates carried the $b / a_{\mathrm{CMY}} 2 \mathrm{gene}$ and exhibited a resistance phenotype with resistance only to beta-lactam antibiotics. The remaining isolate $1773 / 80$ carried the $b / a_{C T X}-\mathrm{M}-1$ gene. However, the plasmid Inc I1/FIB carrying this resistance gene was not transferred to the recipient strain in the conjugation experiments (Table 2). Furthermore, this isolate did not harbor any of the seven APEC virulence genes. In contrast, in the mating experiments, seven of the nine $E$. coli isolates carrying a bla ${ }_{\mathrm{CMY}-2}$ gene were able to transfer the corresponding bla $\mathrm{CMY}_{-2}$-bearing plasmid to the recipient strain. It was shown that isolate $1773 / 50$ carried a $95 \mathrm{~kb}$ conjugative plasmid, replicon type Inc I1/ FIB, with the bla $\mathrm{CMY}_{2}$ gene and additional $h l y$, iroN, iss, ompT and $c v a C$ virulence genes (Table 2). The other conjugative plasmids were either of replicon type I1 or I1/FIB but did not carry any of the virulence genes tested. The conjugation efficiency of these Incl or Incl/FIB type plasmids was moderate to high for isolates $1773 / 7,1773 / 67$ and $1775 / 17\left(3.36 \times 10 \rrbracket^{3}\right.$ to $\left.2.66 \times 10 \rrbracket^{1} \mathrm{cfu}\right)$, while for the rest of the isolates the conjugation transfer was lower (Table 2). 
Table 2

Plasmid mediated resistance to extended-spectrum cephalosporin antibiotics in E. coli isolates from black-headed gulls

\begin{tabular}{|c|c|c|c|c|c|c|c|c|c|c|c|}
\hline \multicolumn{5}{|c|}{ Characterization of $E$. coli-donors } & \multicolumn{7}{|c|}{ Characterization of $E$. colitransconjugants } \\
\hline $\begin{array}{l}\text { Isolate } \\
\text { No }\end{array}$ & Resistotype & Resist. genes & $\begin{array}{l}\text { Virulence } \\
\text { genes }\end{array}$ & $\begin{array}{l}\text { Sequen. } \\
\text { type }\end{array}$ & $\begin{array}{l}\text { Phyl. } \\
\text { group }\end{array}$ & $\begin{array}{l}\text { Inc } \\
\text { group }\end{array}$ & $\begin{array}{l}\text { Resistotype } \\
\text { TR }\end{array}$ & $\begin{array}{l}\text { Resist. } \\
\text { gene } \\
\text { transfer }\end{array}$ & $\begin{array}{l}\text { Efficacy } \\
\text { of } \\
\text { conjugation }\end{array}$ & $\begin{array}{l}\text { Virulence } \\
\text { gene } \\
\text { transfer }\end{array}$ & $\begin{array}{l}\text { Plasn } \\
\text { size } \\
(\mathrm{kb})\end{array}$ \\
\hline $1773 / 7$ & $\begin{array}{l}\text { AMC, AMP, } \\
\text { CPD, CAZ, } \\
\text { CTX, FOX }\end{array}$ & $b / a_{\mathrm{CMY}-2}$ & - & ST38 & $\mathrm{D}$ & 11 & $\begin{array}{l}\text { AMC, AMP, } \\
\text { CPD, CTX, } \\
\text { CAZ, FOX, }\end{array}$ & $b / a_{\mathrm{CMY}-2}$ & $3.36 \times 10^{-3}$ & - & 95 \\
\hline $22 a$ & $\begin{array}{l}\text { AMC, AMP, } \\
\text { CPD, CAZ, } \\
\text { CTX, FOX }\end{array}$ & $b / a_{\mathrm{CMY}-2}$ & - & & $\mathrm{D}$ & $\begin{array}{l}\text { I1, } \\
\text { FIB }\end{array}$ & - & - & - & - & \\
\hline $1773 / 47$ & $\begin{array}{l}\text { AMC, AMP, } \\
\text { CPD, CAZ, } \\
\text { CTX, FOX }\end{array}$ & $b / a_{\mathrm{CMY}-2}$ & $\begin{array}{l}\text { hlyF, } \\
\text { ompt }\end{array}$ & ND* & $\mathrm{D}$ & 11 & $\begin{array}{l}\text { AMC, AMP, } \\
\text { CPD,CAZ,CTX, } \\
\text { FOX }\end{array}$ & $b / a_{\mathrm{CMY}-2}$ & $2.81 \times 10^{-5}$ & - & 110 \\
\hline $1773 / 50$ & $\begin{array}{l}\text { AMC, AMP, } \\
\text { CPD, CTX, } \\
\text { FOX, CIP, } \\
\text { NAL, TET }\end{array}$ & $\begin{array}{l}b / a_{\mathrm{CMY}-2}, b l a_{\mathrm{TEM}-1} \\
\text { tetA }\end{array}$ & $\begin{array}{l}\text { hly, iroN, } \\
\text { iss, } \\
\text { ompT, } \\
\text { cvaC }\end{array}$ & ST2307 & $\mathrm{D}$ & $\begin{array}{l}\text { I1, } \\
\text { FIB }\end{array}$ & $\begin{array}{l}\text { AMC, AMP, } \\
\text { CPD, CAZ, } \\
\text { CTX, FOX }\end{array}$ & $b / a_{\mathrm{CMY}-2}$ & $1.27 \times 10^{-6}$ & $\begin{array}{l}\text { hly, iroN, } \\
\text { iss, } \\
\text { ompT, } \\
\text { cvaC }\end{array}$ & 95 \\
\hline $1773 / 52$ & $\begin{array}{l}\text { AMC, AMP, } \\
\text { CPD, CAZ, } \\
\text { CTX, CIP, } \\
\text { NAL, TET, } \\
\text { FOX }\end{array}$ & $\begin{array}{l}b / a_{\mathrm{CMY}-2}, b / a_{\mathrm{TEM}-1} \\
\text { tet } A\end{array}$ & $\begin{array}{l}\text { iroN, } \\
\text { hlyF, iutA, } \\
\text { iss,ompT, } \\
\text { eitC }\end{array}$ & ST224 & B2 & $\begin{array}{l}\text { I1, } \\
\text { FIB }\end{array}$ & $\begin{array}{l}\text { AMC, AMP, } \\
\text { CPD, CAZ, } \\
\text { CTX, FOX }\end{array}$ & $b / a_{\mathrm{CMY}-2}$ & $3.33 \times 10^{-4}$ & - & $\begin{array}{l}40,11 \\
140\end{array}$ \\
\hline $1773 / 67$ & $\begin{array}{l}\text { AMC, AMP, } \\
\text { CPD, CAZ, } \\
\text { CTX, FOX }\end{array}$ & $b / a_{\mathrm{CMY}-2}$ & iroN & ND* & B1 & $\begin{array}{l}\text { I1, } \\
\text { FIB }\end{array}$ & $\begin{array}{l}\text { AMC, AMP, } \\
\text { CPD, CAZ, } \\
\text { CTX, FOX }\end{array}$ & $b / a_{\mathrm{CMY}-2}$ & $4.2 \times 10^{-2}$ & - & 90 \\
\hline $1773 / 75$ & $\begin{array}{l}\text { AMC, AMP, } \\
\text { CPD, CAZ, } \\
\text { CTX, FOX }\end{array}$ & $b / a_{\mathrm{CMY}-2} b / a_{\mathrm{TEM}-1}$ & $\begin{array}{l}\text { iroN, } \\
\text { hlyF, iutA, } \\
\text { iss,ompT, } \\
\text { eitC, } \\
\text { cvaC }\end{array}$ & ST162 & B2 & $\begin{array}{l}\text { I1, } \\
\text { FIB }\end{array}$ & $\begin{array}{l}\text { AMC, AMP, } \\
\text { CPD, CAZ, } \\
\text { CTX, FOX }\end{array}$ & $b / a_{\mathrm{CMY}-2}$ & $3.3 \times 10^{-5}$ & - & $95,1 \varepsilon$ \\
\hline $1773 / 80$ & $\begin{array}{l}\text { AMP, CPD, } \\
\text { CTX }\end{array}$ & $b / a_{\mathrm{CTX}-\mathrm{M}-1}, b / a_{\mathrm{TEM}-1}$ & - & & B2 & $\begin{array}{l}\text { I1, } \\
\text { FIB }\end{array}$ & - & - & - & - & \\
\hline $1774 / 1$ & $\begin{array}{l}\text { AMC, AMP, } \\
\text { CPD, CAZ, } \\
\text { CTX, FOX, } \\
\text { NAL, TET }\end{array}$ & $\begin{array}{l}b / a_{\mathrm{CMY}-2}, b / a_{\mathrm{TEM}-1} \\
\text { tet } A\end{array}$ & $\begin{array}{l}\text { iroN, } \\
\text { hlyF, iutA, } \\
\text { iss, } \\
\text { ompT, } \\
\text { cvaC }\end{array}$ & & B2 & $\begin{array}{l}\text { I1, } \\
\text { FIB }\end{array}$ & - & - & - & - & \\
\hline $1775 / 17$ & $\begin{array}{l}\text { AMC, AMP, } \\
\text { CPD, CAZ, } \\
\text { CTX, FOX }\end{array}$ & $b / a_{\mathrm{CMY}-2} b / a_{\mathrm{TEM}-1}$ & - & ST34 & B2 & $\begin{array}{l}\text { I1, } \\
\text { FIB, Y }\end{array}$ & $\begin{array}{l}\text { AMC, AMP, } \\
\text { CPD, CAZ, } \\
\text { CTX, FOX }\end{array}$ & $b / a_{\mathrm{CMY}-2}$ & $2.66 x^{10-1}$ & - & 95 \\
\hline
\end{tabular}

*ND, ST designations could not be attributed to isolates E. coli 1773-47 and 1773-67 (Supplementary Table 2)

Phylogenetic analysis was carried out on all the isolates conferring resistance to extended-spectrum cephalosporins. Five isolates belonged to the phylogenetic-group B2, four isolates belonged to group D and one isolate was assigned to the B1 group. Five different sequence types (ST38, ST2307, ST224, ST162 and ST34) were detected in these E. coli isolates.

\section{Resistance to fluoroquinolones (FQ)}

In this study, high-level resistance to fluoroquinolones was detected in ten isolates. The MIC values in FQ resistant strains ranged from 4 to 32 mg/L (Table 3 ). Mutations in the quinolone resistance determining region (QRDR) of the topoisomerase genes were investigated in a few selected isolates (Table 3 ). For these isolates it was shown that high MIC values of CIP were achieved due to multiple mutations in the gyrA, parC and/or parE genes (Table 3), while single point mutations in the gyrA gene were detected in isolates resistant to NAL, resulting in amino acid transitions Ser83 $\rightarrow$ Leu or Asp87 $\rightarrow$ Asn. Plasmid-mediated resistance (PMQR) determinants were not found.

Table 3: Mutations in topoisomerase genes in representative $E$. coli isolates from blackheaded gulls 


\begin{tabular}{|lllll|}
\hline Isolate No & gyrA & parC & parE & MIC-CIP \\
& & & & mg/L \\
\hline $1773 / 1$ & Ser83Leu, Asp87Asn & $/$ & $/$ & 4 \\
\hline $1773 / 6$ & Ser83Leu, Asp87Asn & Ser80lle & $/$ & 4 \\
\hline $1773 / 25$ & Ser83Leu, Asp87Asn & Ser80lle & $/$ & 8 \\
\hline $1773 / 70$ & Ser83Leu, Asp87Asn & Ser80lle & $/$ & 4 \\
\hline $1773 / 87$ & Ser83Leu, Asp87Asn & Ser80lle & Leu416Phe & 16 \\
\hline 17 & Ser83Leu, Asp87Asn & Ser80lle & Ser458Ala & 16 \\
\hline $1773 / 50$ & Ser83Leu Asp87Asn & $/$ & $/$ & 32 \\
\hline $1773 / 52$ & Ser83Leu Asp87Asn & Ser80lle & Ser458Ala & 32 \\
\hline $1773 / 86$ & Ser83Leu & $/$ & $/$ & 0.25 \\
\hline 16 & Asp87Tyr & $/$ & $/$ & 0.125 \\
\hline
\end{tabular}

PMQR genes ( $q n r S$, qnrA, qnrB, qnrC, qnrD, aac-lb-cr,qepA, oqxA, oqxB) were not identified in $E$. coli isolates. Sequencing of the QRDR was not done in isolates $1774 / 18$ and 1776/13 resistant to CIP and in isolates resistant to NAL (1773/59, 1774/1, 1774/14, 1774/18, 1774/25, and 1776/4).

\section{Discussion}

In the course of the present study, it was shown that almost half of the black-headed gulls living in the wild carried resistant or multi-resistant $E$. coli. The resistance patterns, but also the resistance genes detected in the $E$. coli isolates were similar to those of other research studies, in which fecal samples from waterfowl were taken (Dolejska et al., 2007; Poeta et al., 2008; Dolejska et al., 2009; Literak et al., 2010; Tausova et al., 2012). However, the significant resistance to fluoroquinolone in $E$. coli isolates from gulls in Serbia implies the overuse of these antibiotics in human and veterinary medicine and the unsafe disposal of communal and medical waste in Serbia.

In the present collection of isolates, resistance to extended-spectrum cephalosporins was mediated by the plasmid-borne resistance gene bla $\mathrm{CMY}_{2}$ except in one case where the bla $a_{\mathrm{CTX}-\mathrm{M}-1}$ gene was identified. Often the CMY-2 plasmid carriers were resistant only to beta-lactam antibiotics. A similar result was obtained in another study, in which only three out of eight cephalosporin-resistant $E$. coli isolates with a bla $\mathrm{CMY}_{2}$ gene from gulls and bald eagles from Alaska were multidrug-resistant (Ahlstrom et al., 2018). However, in E. coli isolates from food-producing animals, the bla ${ }_{\mathrm{CTX}-\mathrm{M}-1}$ gene is widely distributed in the Mediterranean area (Dandachi et al., 2018). The CTX-M family is also prevalent in Enterobacteriaceae in many European countries, mainly due to the epidemic spread of resistance plasmids. It is important that CTX-M carriers were found not only in E. coli and Klebsiella pneumoniae isolates from hospital patients but also in patients with community-acquired infections (D'Andrea et al., 2013; Canton et al., 2014). The bla $\mathrm{CTX}_{\mathrm{M}-\mathrm{M}}$ gene has also been detected in $E$. coli isolates from Serbia from cases of clinical bovine mastitis, from diseased pigs and wildlife (Todorović et al., 2018; Velhner et al., 2018), and in this work also in an isolate from a gull.

We detected several important virulence genes in the $E$. coli isolates conferring resistance to beta-lactam antibiotics. These virulence genes comprised genes encoding a siderophore receptor for the iron acquisition mechanism (iro $N$ ), an episomal outer membrane protease (omp $T)$, a putative avian hemolysin ( $h / y)$, a serum survival protein (iss) and the colicin $\mathrm{V}$ structural gene $(\mathrm{cvaC})$, which is important for increasing the adhesion and invasiveness of $E$. coli and other species of the Enterobacteriaceae family and which are frequently identified in avian pathogenic E. coli-APEC isolates (Gilson et al., 1987; Johnson et al., 2008; Johnson et al., 2010).

In the analyzed collection of isolates, only those with the CMY plasmid carried APEC virulence genes. Our results are therefore similar to those presented in the

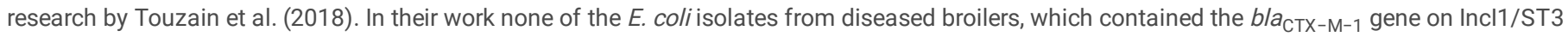
conjugative plasmids, carried APEC virulence genes. However, virulence genes were found on bla $\mathrm{CMY}-2_{2}$ plasmid of the IncF replicon type (Touzian et al., 2018).

The genotypes of the ESBL-producing E. coli from gulls living in the vicinity to a dense human population were similar to the genotypes found in human isolates in southern France (Bonnedahl et al., 2009) and Sweden (Bonnedahl et al., 2010). Therefore, E. coli from gulls can serve as a biological indicator of environmental contamination due to their habits of living near humans and feeding on landfills along or off the coast (Bonnedahl et al., 2009; Bonnedahl et al., 2014).

Twelve E. coli isolates from gulls in Barrow, Alaska were of sequence type ST38 and carried the bla $a_{\mathrm{CTX}-\mathrm{M}-14}$ gene (Bonnedahl et al., 2014). In this work only isolate 1773/7 was identified as sequence type 38 and this carried the Incl1/CMY plasmid. In the European Union, the ST38 lineage is considered typical for poultry but has also been found in human $E$. coli isolates. It was evident that in $E$. coli isolates from Germany IncK, Incl and IncA/C plasmids most frequently carry the bla $\mathrm{CMY}_{-2}$ gene, while IncA/C plasmids were the most common CMY carriers in North America (Pitech et al., 2018). Horizontal transfer via plasmids is perhaps the most likely mechanism of dissemination of the bla $\mathrm{CMY}_{\mathrm{C}-2}$ gene although the epidemiological spread of specific lineages such as $\mathrm{CMY}-2$ producing ST131 is involved in the transmission of CMY plasmid as well. Nevertheless, a common ancestor of $E$. coli isolates carrying the $b l a_{\mathrm{CMY}-2}$ gene has been identified in genetically distant strains suggesting that bacteria change over time due to their genome plasticity and diversity (Pitech et al., 2018). It was found that the successes in the proliferation of CMY-2 plasmids depend on the selective pressure posed by the use of antibiotics or on the size of the plasmid since 
larger plasmids, such as CMY carriers, are associated with a significant fitness cost (Subbiah et al., 2011). Therefore, the long-term stability of the bla $\mathrm{CMY}_{2}$ plasmids in E. coli isolates from gulls, which are discussed in this study, should be determined in the future using in vitro experimental approaches.

In this work, we detected high-level resistance to FQ in several isolates. However, resistance to FQ was also observed in E. coli isolates from mallards and hearing gulls residing at the Polish coast of the Baltic sea (Literak et al., 2010) and from feces of gulls, pigeons and birds of prey in Portugal, Sweden and Spain (Vredenburg et al., 2013). Resistance to FQ antibiotics and extended-spectrum cephalosporins was also recently found in extra-intestinal pathogenic $E$. coli (ExPEC) isolates from silver gulls residing at the coastline in Australia which included pandemic ExPECST131 strains belonging to clade C (C1-H30-R and C2-H30-Rx), (Mukerji et al., 2019).

Resistance to beta-lactam antibiotics and fluoroquinolones in bacteria isolated from wild animals, including wild birds, is of concern because the environment appears to be contaminated by anthropogenic activities, affecting the bacterial flora of wildlife (Mukerji et al., 2019). While ESBL-producing E. coli isolates from food-producing animals are often multi-resistant due to co-selection mechanisms (Michael et al., 2017), E. coli isolates from black-headed gulls carrying the plasmid-borne AmpC gene ( bla $_{\mathrm{CMY}-2}$ ) appear to be resistant only to beta-lactam antibiotics (Atterby et al., 2016; Touzian et al., 2018). It must also be pointed out that the global spread of epidemic $E$. coli clonal lineages is of great importance in medicine and the spread of such isolates needs to be closely monitored (Pitout and DeVinney, 2017).

\section{Conclusion}

In conclusion, multidrug resistance in E. coli isolates from black-headed gulls residing in the city of Novi Sad was detected in 22 out of 96 isolates. However, five isolates which were resistant only to beta-lactam antibiotics carried the bla $\mathrm{CMY}_{2}$ gene on transferable plasmids. Virulence genes ( $h / y$, iroN, iss, ompT and $\mathrm{cvaC}$ ) were detected on one conjugative CMY plasmid as well. In isolates with high resistance to fluoroquinolones, the resistance was due to multiple mutations in the topoisomerase genes. This result may have been caused by environmental contamination in Serbia.

\section{Declarations}

\section{Author contribution}

MV, BJ and MK designed the study and analyzed the results, DT, KN and GL performed the sampling and the experiments, MV and BJ wrote the manuscript, CK reviewed the manuscript.

\section{Disclosure statement}

No competing financial interest exists.

\section{Funding Information}

The research was funded by the grant from the Ministry of Education, Science and Technological Development of the Republic of Serbia, by the Contract of implementation and financing of scientific research work of NIV-NS, Contract No. 451-03-68/2020-14/200031 and by the Contract of implementation and financing of scientific research work of IMGGE, Contract No. 451-03-68/2020-14/ 200042.

\section{References}

Admassie M (2018) Current review on molecular and phenotypic mechanisms of bacterial resistance to antibiotic. Sci J Clin Med 7:13-19.

Ahlstrom CA, Bonnedahl J, Woksepp H, Hernandez J, Olsen B, Ramey AM (2018) Acquisition and dissemination of cephalosporin-resistant E. coli in migratory birds sampled at an Alaska landfill as inferred through genomic analysis. Sci Rep 8:7361, http://doi:10.1038/s41598-018-25474-w.

D’Andrea MM, Arena F, Pallecchi L, Rossolini GM (2013) CTX-M type $\beta$-lactamases: A successful story of antibiotic resistance. Int J Med Microbiol 303:305317.

Atterby C, Ramey AM, Hall GG, Järhult J, Börjesson S, Bonnedahl J (2016) Increased prevalence of antibiotic-resistant E. coli in gulls sampled in Southcentral Alaska is associated with urban environments. Infect Ecol Epidemiol 6:32334-http://dx.doi.org/10.3402/iee.v6.32334.

Atterby C, Börjesson S, Ny S, Järhult JD, Byfors S, Bonnedahl J (2017) ESBL-producing Escherichia coli in Swedish gulls-a case of environmental pollution from humans? PLoS ONE, 12(12): e0190380. https://doi.org/10.1371/journal.pone.0190380.

Bonnedahl J, Drobni M, Gauthier-Clerc M, Hernandez J, Granholm S, Kayser Y, Melhus A, Kahlmeter G, Waldenstörm J, Johansson A, Olsen B (2009) Dissemination of Escherichia coli with CTX-M type ESBL between humans and yellow-legged gulls in the south of France. PLoS ONE 4(6): e5958. https://doi:10.1371/journal.pone.0005958.

Bonnedahl J, Drobni P, Johansson A, Hernandez J, Melhus A, Stedt J, Olsen B, Drobni M (2010) Characterization, and comparison, of human clinical and blackheaded gull (Larus ridibundus) extended-spectrum beta-lactamase-producing bacterial isolates from Kalmar, on the southeast coast of Sweden. $\mathrm{J}$ Antimicrob Chemother 65:1939-1944 
Bonnedahl J, Hernandez J, Stedt J, Waldenström J, Olsen B, Drobni M (2014) Extended-spectrum ß-lactamases in Escherichia coli and Klebsiella pneumoniae in gulls, Alaska, USA. Emerg Infect Dis 20:897-899.

Canton R, Novais A, Valverde A, Machado E, Peixe L, Baquero F, Coque TM (2008) Prevalence and spread of extended-spectrum $\beta$-lactamase producing Enterobacteriaceae in Europe Clin Microbil Infect 14:144-153.

Clinical and Laboratory Standards Institute, Methods for dilution antimicrobial susceptibility tests for bacteria that grow aerobically; Approved standard-tenth edition, CLSI document M07, 11 $11^{\text {th }}$ ed. Wayne, PA, USA, 2018.

Clinical and Laboratory Standards Institute, Performance standards for antimicrobial susceptibility testing; Twenty-fifth Informational Supplement. CLSI document M100, 28 ${ }^{\text {th }}$ ed. Wayne, PA, USA, 2018.

Dandachi I, Chabou S, Daoud Z, Rolain JM (2018) Prevalence and emergence of extended-spectrum cephalosporin-carbapenem-and colistin-resistant gram negative bacteria of animal origin in the Mediterranean basin. Front Microbiol 9:2299. https://

doi:10.3389/fmicb.2018.02299.

Dolejska M, Cizek A, Literak I (2007) High prevalence of antimicrobial-resistant genes and integrons in Escherichia coli isolates from black-headed gulls in the Czech Republic. J Appl Microbiol 103:11-19.

Dolejska M, Bierošová B, Kohutová L, Literák I, Čižek A (2009) Antibiotic-resistant Salmonella and Escherichia coli isolates with integrons and extendedspectrum beta-lactamases in surface water and sympatric black-headed gulls. J Appl Microbiol 106: 1941-1950.

The European Union summary report on antimicrobial resistance in zoonotic and indicator bacteria for humans, animals and food in 2017. EFSA Journal 2019;17(2):5598, 2778 pp. https://doi.org/10.2903/j.efsa.2019.5598 (page 41).

Faccone D, Moredo FA, Giacoboni GI, Albornoz E, Alarcón L, Nievas VF, Corso A (2019) Multidrug-resistant Escherichia coli harbouring mcr-1 and bla genes isolated from swine in Argentina. J Glob Antimicrob Resist 18:160-162.

Gilson L, Mahanty HK, Kolter R, (1987) Four plasmid genes are required for colicin V synthesis export, and immunity. J Bacteriol 169:2466-2470.

Grenni P, Ancona V, Caracciolo AB, (2018) Ecological effects of antibiotics on natural ecosystems: A review. Microchem J 136:25-39.

Gwozdzinski K, Azarderakhsh S, Imirzalioglu C, Falgenhauer L, Chakraborty T (2018) An improved medium for colistin susceptibility testing. J Clin Microbiol 56(5),56:e01950-17. https://doi.org/10.1128/JCM .01950-17.

Hernandez J, Johansson A, Stedt J, Bengtsson S, Porczak A, Granholm S, González-Acuña Olsen B, Bonnedahl J, Drobni M (2013) Characterization and comparison of extended-spectrum $\beta$-lactamase (ESBL) resistance genotypes and population structure of Escherichia coli isolated from Franklin's gulls (Leucophaeus pipixcan) and humans in Chile. PLoS ONE 8(9):e76150. doi:10.1371/journal.pone.0076150.

Johnson TJ, Wannemuehler Y, Doetkott C, Johnson SJ, Rosenberger SC, Nolan LK (2008) Identification of minimal predictors of avian pathogenic Escherichia coli virulence for use as a rapid diagnostic tool. J Clin Microbiol 46:3987-3996.

Johnson TJ, Thorsness JL, Anderson CP, Lynne AM, Foley SL, Han J, Fricke WF, McDermott PF, White DG, Khatri M, Stell AL, Flores C, Singer RS (2010) Horizontal gene transfer of ColV plasmid has resulted in a dominant avian clonal type of Salmonella enterica serovar Kentucky. PLoS ONE, 5(12): e15524. https://doi:10.1371/journal.pone.0015524.

Johnson JR, Nicolas-Chanoine M-H, DebRoy C, Castanheira M, Robicsek A, Hansen G, Weissman S, Urban C, Platell J, Trott D, Zhanel G, Clabots C, Johnston BD, Kuskowski MA and the MASTER investigators. Comparison of Escherichia coli ST131 pulsotypes, by epidemiologic traits, 1997-2009. 2012. Emerg Infect Dis 18:598-607.

Jovcic B, Lepsanovic Z, Suljagic V, Rackov G, Begovic J, Topisirovic L, Kojic M (2011) Emergence of NDM-1 metallo- $\beta$-lactamase in Pseudomonas aeruginosa clinical isolates from Serbia. Antimicrob Agents Chemother 55:3929-3931.

Levy SB, Marshall B (2004) Antibacterial resistance worldwide: causes, challenges and responses. Nat Med 10, (12 Suppl), S122-S129.

Literak I, Dolejska M, Janoszowska D, Hrusakova J, Meissner W, Rzyska H, Bzoma S, Cizek A (2010) Antibiotic-resistant Escherichia coli bacteria, including strains with genes encoding the extended-spectrum beta-lactamase and QnrS, in waterbirds on the Baltic Sea coast of Poland. Appl Environ Microbiol $76: 8126-8134$.

McDaniels AE, Rice EW, Reyes AL, Johnson CH, Haugland RA, Stelma GN Jr. (1996) Conformational identification of Escherichia coli, a comparison of genotype and phenotype assays for glutamate decarboxylase and beta-D-glucuronidase. Appl Environ Microbiol 62:3350-3354.

Michael GB, Kaspar H, Siqueira AK, Costa EF, Corbellini LG, Kadlec K, Schwarz S (2017) Extended-spectrumß-lactamase (ESBL)-producing Escherichia coli isolates collected from disease food-producing animals in the GERM-Vet monitoring program 2008-2014. Vet Microbiol 200:142-150. 
Mukerji S, Stegger M, Truswell AV, Laird T, Jordan D, Abraham RJ, Harb A, Barton M, O'Dea M, Abraham S (2019) Resistance to critically important antimicrobials in Australian silver gulls (Chroicocephalus novaehollandiae) and evidence of anthropogenic origins. J Antimicrob Chemother 74:2566-2574, https://doi:10.1093/jac/dkz242.

Nicolas-Chanoine M-H, Bertrand X, Madec J-Y (2014) Escherichia coli ST131, an intriguing clonal group. Clin Microbiol Rev 27:543-574.

Parker D, Sniatynski MK, Mandrusiak D, Rubin JE (2016) Extended-spectrum ß-laactamase producing Escherichia coli isolated from wild birds in Saskatoon, Canada. Lett Appl Microbiol 63:11-15.

Phornphisutthimas S, Thamchaipenet A, Panijpan B (2007) Conjugation in Escherichia coli. Biochem Mol Biol Edu 35:440-445.

Pietsch M, Irrgang A, Roschanski N, Michael GB, Hamprecht A, Rieber H, Käsbohrer A, Schwarz S, Rösler U, Kreienbrock L, Pfeifer Y, Fuchs S, Werner G, and RESET study group, 2018. Whole genome analysis of CMY-2 producing Escherichia coli isolates from humans, animals and food in Germany. BMC Genomics 19:601, https://doi.org/10.1186/s12864-018-4976-3.

Pitout JDD, DeVinney R (2017) Escherichia coli ST131: a multidrug-resistant clone primed or global domination. F1000Research 2017, 6(F1000 Faculty Rev):195 (doi: 10.12688/f1000research.10609.1).

Poeta P, Radhouani H, Igrejas G, Gonçalves A, Carvalho C, Rodrigues J, Vinué L, Somalo S, Torres C (2008) Seagulls of the Berlengas natural reserve of Portugal as carriers of fecal Escherichia coli harboring CTX-M and TEM extended-spectrum beta-lactmases. Appl Environ Microbiol 74:7439-7441.

Poirel L, Potron A, De La Cuesta C, Cleary T, Nordmann P, Munoz-Price SL (2012) Wild coastline birds as reservoirs of broad-spectrum- $\beta$-lactamase producing Enterobacteriaceae in Miami Beach, Florida. Antimicrob Agents Chemother 56:2756-2758.

Ramey AM, Hernandez J, Tyrlöv V, Uher-Koch BD, Schmutz JA, Atterby C, Järhult JD, Bonnedahl J (2018) Antibiotic-resistant Escherichia coli in migratory birds inhabiting remote Alaska. EcoHealth 15, 72-81, https://doi.org/10.1007/s10393-017-1302-5.

Schwarz S, Silley P, Simjee S, Woodford N, van Duijkeren E, Johnson AP, Gaastra W (2010) Editorial: assessing the antimicrobial susceptibility of bacteria obtained from animals.

J Antimicrob Chemother 65:601-604.

Shabana II, Al-Enazi AT (2020) Investigation of plasmid-mediated resistance in E. coli isolated from healthy and diarrheic sheep and goats. Saudi J Biol Sci 27:788-796.

Simões RR, Poirel L, Da Costa PM, Nordmann P (2010) Seagulls and beaches as reservoirs for multidrug-resistant Escherichia coli. Emerg Infec Dis 16:110112.

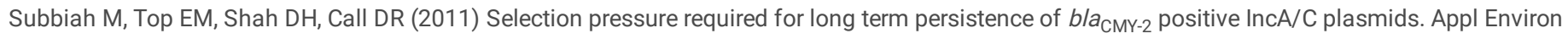
Microbiol 77:4486-4493.

Tausova D, Dolejska M, Cizek A, Hanusova L, Hrusakova J, Svoboda O, Camlik G, Literak I (2012) Escherichia coli with extended-spectrum $\beta$-lactamase and plasmid-mediated quinolone resistance genes in great cormorants and mallards in Central Europe. J Antimicrob Chemoth 67:1103-1107.

Todorović D, Velhner M, Grego E, Vidanović D, Milanov D, Krnjaić D, Kehrenberg C (2018) Molecular characterization of multidrug-resistant Escherichia coli isolates from bovine mastitis and pigs in the Vojvodina Province, Serbia. Microb Drug Resist 24:95-103.

Touzain F, Le Devendec L, de Boisséson C, Baron S, Jouy E, Perrin-Guyomard A, Blanchard Y, Kempf I (2018) Characterization of plasmid harboring bla ${ }_{C T X-M}$ and bla ${ }_{\mathrm{CMY}}$ genes in E. coli from French broilers. PLoS ONE 13(1):e0188768. https://doi.org/10.1371/journal.pone.0188768.

Vandecraen J, Chandler M, Aertsen A, Van Houdt R (2017) The impact of insertional sequence on bacterial genome plasticity and adaptability. Crit Rev Microbiol 43:

709-730.

Velhner M, Todorović D, Grego E, Jovčić B, Prunić B, Stojanov I, Kehrenberg C (2018) Fluoroquinolone-resistant and extended-spectrum beta-lactamase producing Escherichia coli isolates from free-living wild animals. Vet Microbiol 223:168-172.

Vredenburg J, Varela AR, Hasan B, Bertilsson S, Olsen B, Narciso-da-Rocha C, Bonnedahl J, Stedt J, Da Costa PM, Manaia CM (2013) Quinolone-resistant Escherichia coli isolated from birds of prey in Portugal are genetically distinct from those isolates from water environments and gulls in Portugal, Spain and Sweden. Environ Microbiol 16:995-1004, doi:10.1111/1462-2920.12231.

Wellington EMH, Boxall ABA, Cross P, Feil EJ, Gaze WH, Hawkey PM, Johnson-Rollings AS, Jones DL, Lee NM, Otten W, Thomas CM, Williams AP (2013) The role of the natural environment in the emergence of antibiotic resistance in Gram-negative bacteria. Lancet Infect Dis 13:155-165.

Wirth T, Falush D, Lan R, Colles F, Mensa P, Wieler LH, Karch H, Reeves PR, Maiden MCJ, Ochman H, Achtman M (2006) Sex and virulence in Escherichia coli: an evolutionary perspective. Molec Microbiol 60:1136-1151. 
Zurfluh K, Albini S, Mattmann P, Kindle P, Nüesch-Inderbinen M, Stephan R, Vogler BR (2019) Antimicrobial resistant and extended-spectrum $\beta$-lactamase producing Escherichia coli in common wild bird species in Switzerland. MicrobiologyOpen 2019;8:e845. https://doi.org/10.1002/mbo3.845.

\section{Supplementary Files}

This is a list of supplementary files associated with this preprint. Click to download.

- SupplementaryTables1.docx

- SupplementaryTableS2.docx 